\title{
A novel case of prolonged Ifosfamide encephalopathy and long-term treatment with methylene blue: a case report and review of literature
}

\author{
Gabriel Chain ${ }^{{ }^{*}(\mathbb{D}}$, Mudit Kalia $^{1}$, Karen Kestenbaum$^{2}$, Lara Pappas ${ }^{2}$, Anna Sechser-Perl², \\ Gadi Abebe Campino ${ }^{3}$ and Nibal Zaghloul ${ }^{1,2}$
}

\begin{abstract}
Background: Encephalopathy following Ifosfamide treatment is a well-described phenomenon that is typically treated with Methylene Blue (MB). Chloroacetaldehyde, a potentially neurotoxic metabolite of Ifosfamide is hypothesized to cause this encephalopathy. Current guidelines for treatment is to stop Ifosfamide and provide supportive care. MB acts to inhibit Chloroacetaldehyde formation and has been described as a therapy and prophylaxis for Ifosfamide-encephalopathy. MB is effective within 30 min and lasts up to 3 days. Prolonged encephalopathy and MB therapy has not been described in the literature as lasting longer than 30 days following treatment.

Case presentation: We present the case of an 11-year-old female with autistic spectrum disorder and recurrent episodes of severe somnolence for 7 months following Ifosfamide therapy for her Non-Germinomatous Germ Cell Tumor (GCT). Periods of somnolence occurred prior to receiving cranial RT. Administration of MB gave immediate but limited response, with resolution of somnolence lasting 1-2 days between administrations. The somnolence could not be explained by neuroimaging or laboratory evaluation, but EEG indicated persistent encephalopathy.

Conclusion: A literature review determines that neurotoxicity is a side effect of Ifosfamide, but this effect has not been described persisting longer than 30 days. Our case continued to require treatment with MB for 7 months following cessation of therapy. We report these novel clinical findings, and hypothesize that there could be a genetic/metabolic component linking this reaction to Ifosfamide with the case patient's pre-existing autism. This possible association may also correlate to the already-established link between autism and the development of GCTs. This hypothesis leads to further discussion on the suitable usage of Ifosfamide in children with co-morbidities and the necessity of screening prior to its usage.
\end{abstract}

Keywords: Somnolence, Ifosfamide, Methylene blue, Encephalopathy

*Correspondence: gabrielschain@gmail.com

1 Department of Pediatrics, The Children's Hospital at Saint Peter's

University Hospital, 254 Easton Avenue, New Brunswick, NJ 08901, USA

Full list of author information is available at the end of the article

\section{Background}

Germ Cell Tumors (GCT) account for 3\% pediatric brain tumors and are subcategorized into Germinomas (G) and Non-Germinomatous (NG) GCT [1]. Most NGGCT involve the pineal or pituitary glands [2]. Pineal tumors often present with symptoms of hydrocephalus including lethargy, vomiting and mental status changes and 
pituitary tumors often present with polyuria or growth disruption.

Ifosfamide is a chemotherapeutic agent, which is a synthetic analog of cyclophosphamide. Side effects of Ifosfamide can include hair loss, bladder irritation and central nervous system (CNS) toxicity [3, 4]. These CNS toxicities include encephalopathy, lethargy and personality changes. During the metabolism of Ifosfamide, Chloroacetaldehyde is produced which is a potentially neurotoxic metabolite that crosses the blood-brain barrier and has been suggested as the etiology behind Ifosfamide encephalopathy [5].

The incidence of CNS toxicity following Ifosfamide therapy is estimated between 10 to $20 \%$ [2]. High doses ( $>$ 2G/M2/Day) of Ifosfamide correlate to higher rates of adverse effects, however, encephalopathy can be demonstrated at any dose and specific blood levels of Ifosfamide have not been clearly linked to the development of neurotoxicity. CNS toxicity usually presents acutely following drug administration and subsequently resolves following drug discontinuation [5]. Certain studies have demonstrated the effects lasting for up to 30 days following treatment [6]. Current guidelines for Ifosfamide encephalopathy include treatment cessation, supportive care and the use of Methylene Blue (MB) as a therapeutic and prophylactic treatment. Risk factors for Ifosfamideencephalopathy include hypoalbuminemia and previous CNS disease [7].

$M B$ is a therapy for Ifosfamide-encephalopathy and research suggests MB's mechanism involves inhibition of monoamine oxidase which is involved in Chloroacetaldehyde formation. Potential adverse effects of MB include discoloration of the skin, vomiting and hemolytic anemia [8]. MB is effective within $30 \mathrm{~min}$ and can last up to 3 days.

\section{Case presentation}

We present the case of an $11 \mathrm{yr}$ female with a background of autism spectrum disorder of moderate severity (which presented in early childhood) who presented with 4 months of cognitive changes. Brain MRI detected a pineal tumor with hydrocephalus and spinal metastasis. GCT markers beta-HCG and alpha-fetoprotein (AFP) were elevated within the Cerebrospinal fluid (CSF). They underwent right frontal VP (ventriculoperitoneal) shunt insertion and tumor pathology reported an NGGCT. She was initiated onto the high-risk treatment arm: COG ACNSO122, which includes 6 cycles of induction chemotherapy (alternating cycles of Carboplatin/Etoposide and Ifosfamide/Etoposide and craniospinal radiation with boost to the tumor bed. Each cycle of Ifosfamide included $1800 \mathrm{mg} / \mathrm{m}^{2} /$ day for 5 days. The patient's baseline physical exam was unremarkable.
During the 4th course of chemotherapy (Ifosfamide/ Etoposide) the patient developed sequelae of Ifosfamide encephalopathy which included drowsiness, confusion and somnolence. As per protocol for Ifosfamide toxicity (grade 2/3), treatment was started with MB (IV, $1 \mathrm{mg} / \mathrm{kg}$, BID). Within hours of initiation, patient orientation returned to baseline. Multidisciplinary discussion was held to discuss the risks/benefit of continued Ifosfamide treatment and a decision was made to continue treatment with close observation and continued use of MB. During the 6th cycle (Ifosfamide/Etoposide, without concurrent use of the anti-emetic Aprepitant), 4 months after treatment initiation, MB was prophylactically used from Day -1 . On day 4 of this cycle, however, the patient developed somnolence. CT and MRI brain were normal, EEG demonstrated mild encephalopathy. Due to concerns of early hydrocephalus, VP shunt revision was performed, however, the somnolence persisted only resolving following MB treatment.

Ifosfamide metabolites, ammonia levels, thiamine levels, lumbar puncture and blood gas were all unchanged from their baseline. AFP/HCG, following initial elevation at diagnosis, then normalized and remained so on serial analysis, EEG continued to demonstrate mild encephalopathy both prior to, and following MB. Levetiracetam was trialed with no benefit noted.

During the radiation course, 7 months following diagnosis, the patient required multiple readmissions/ reviews for recurrent episodes of somnolence. Shunt revision did not alter these episodes. CT and MRI did not note new pathology and serum AFP and B-HCG values continued to be within normal limits. EEG continued to demonstrate encephalopathy. During all these episodes, MB was used and within $30 \mathrm{~min}$ of initiation, the patient was at baseline. Physical exam demonstrated generalized reduced awakening with no other focal neurological findings.

After discussion, radiation therapy was initiated under close observation and no clinical deterioration was reported. Following this course of treatment, the patient was fully alert and orientated. They underwent further neurocognitive testing and they continue to have episodes of recurrent somnolence, however, there were significant cognitive deficits affecting behavior and memory. MRI of brain and spine showed no evidence of disease and blood/CSF markers remained negative. An "Invitae" genetic predisposition work-up was completed (including oncologic panel for germcell tumor predisposition and metabolic panels which included assessments for inborn errors of metabolism), which thus far does not note any significant findings. The patient continues to be in remission without evidence of disease. 


\section{Discussion and conclusion}

Ifosfamide-related encephalopathy is a phenomenon well described in the literature. The first reported case of Ifosfamide encephalopathy and MB treatment was documented in 1996 [9] and describes IE as occurring between $48 \mathrm{~h}$ and 30 days following treatment [10]. Prolonged Ifosfamide encephalopathy and prolonged treatment are novel findings that have not been described in the pediatric population [5]. A case series does describe two reports of prolonged Ifosfamide encephalopathy in an adult population, including a 41 year old woman who developed cognitive dysfunction following Ifosfamide infusion [11]. She was not treated with MB and her neurologic abnormalities persisted beyond 10 years. Encephalopathy can occur at any doses of Ifosfamide, however higher doses do predispose to a higher incidence of adverse effects.

Due to our case patient's diagnosis of autism, we reviewed the literature for any correlation between the diagnosis of autism and the presence or severity of Ifosfamide encephalopathy. Whilst no specific data is published describing this correlation, there is established data associating Autism Spectrum Disorder (ASD) with the development of GCTs [12]. Therefore, we hypothesize that a diagnosis of ASD may also predispose to the toxic effects of Ifosfamide. Similarly, whilst no single physiological pathway is validated for this link, we propose that several plausible theories exist, for example the established effect of Chloroacetaldehyde on Glutathione, imbalance of which is a suggested a pathway to ASD $[13,14]$. This hypothesis may indicate a need for more extensive preliminary screening of comorbid candidates requiring Ifosfamide. We acknowledge that this hypothesis is currently only supported by sparse observational reports, but we hope that this case will prompt others to report similar findings.

We also consider that neurocognitive disorders (such as autism spectrum disorder), may be confounders when analyzing the presentation of 'somnolence.' We note, for example, that autism is a risk factor for sleep disturbance [15] and this can lead to neuropsychiatric complications. Our case patient and caregiver did not report significant sleep disturbance and overnight EEG was not indicative of sleep pathology. Polysomnography may help to qualify these episodes of 'somnolence.'

The neuroprotective effects of $\mathrm{MB}$ was evaluated in a case review of 52 patients, which concluded that $\mathrm{MB}$ is an effective treatment for Ifosfamide encephalopathy [16]. The study also demonstrated that prophylactic treatment with MB leads to fewer and milder cases of Ifosfamide encephalopathy [17]. The mechanism of Ifosfamide encephalopathy centers around a metabolite, Chloroacetaldehyde, which is hypothesized to be inhibited via treatment with MB. Chloroacetaldehyde is typically eliminated within a $24-\mathrm{h}$ period [18]. To investigate if delayed elimination of Chloroacetaldehyde is causing the observed symptoms, a metabolome would need to be performed on the patient. If metabolic signatures of Chloroacetaldehyde are identified following cessation of Ifosfamide, this would support the hypothesis that prolonged Ifosfamide encephalopathy is caused by increased retention of Chloroacetaldehyde. Blood levels of Ifosfamide in our patient were noted to be normal, however specific levels have not been validated when linked to encephalopathy.

In conclusion, Ifosfamide-induced encephalopathy is a well-described toxicity which usually lasts for a period of days to weeks following treatment. MB is described as a therapy for this encephalopathy, but prolonged encephalopathy and treatment with $\mathrm{MB}$ after several months of cessation of treatment with Ifosfamide has not been previously reported. We highlight this case in order contribute to the ongoing research on the usage of Ifosfamide in children with co-morbidities and the potential value of genetic or metabolic screening prior to its use.

\section{Abbreviations}

MB: Methylene Blue; GCT: Germ Cell Tumor; G: Germinomatous; NG: Non-Germinomatous; CNS: Central Nervous System; CSF: Cerebrospinal Fluid; VP: Ventriculoperitoneal; ASD: Autism Spectrum Disorder; EEG: Electroencephalogram.

\section{Acknowledgements \\ This research continues on work that was accepted as an abstract/poster to the 2020 American Society of Pediatric Hematology/Oncology (cancelled due to COVID-19). Lawren Wilkins, Case patient and patient's family, Mr. C Chain \& Professor B Chain, Tel HaShomer Hospital, Israel, Procure Radiation Centre (New Jersey, USA), The Children's Hospital at Saint Peter's University Hospital.}

\section{Authors' contributions}

GC \& MK drafted and formatted this report. AS, LP, KK, NZ and KK were part of the patient's treatment team and were involved in editing this report. All authors read and approved the final manuscript.

\section{Funding}

No funding was used for this report.

\section{Availability of data and materials}

The datasets used and/or analyzed during the current study are available from the corresponding author on reasonable request.

\section{Declarations}

Ethics approval and consent to participate Not Applicable.

\section{Consent for publication}

Written informed consent was obtained from the patient's parent for publication of this case report and any accompanying images. A copy of the written consent is available for review by the Editor of this journal.

\section{Competing interests}

The authors declare that they have no competing interests. 


\section{Author details}

'Department of Pediatrics, The Children's Hospital at Saint Peter's University Hospital, 254 Easton Avenue, New Brunswick, NJ 08901, USA. ${ }^{2}$ Division of Pediatric Hematology/Oncology, The Children's Hospital at Saint Peter's University Hospital, New Brunswick, NJ 08901, USA. ${ }^{3}$ Pediatric Hemato-Oncology division, Safra Children's Hospital, Sheba Medical Center, Ramat Gan, Israel.

Received: 6 September 2021 Accepted: 27 January 2022

Published online: 02 February 2022

\section{References}

1. Packer RJ, Cohen BH, Cooney K. Intracranial germ cell tumors. Oncologist. 2000;5(4):312-20. Erratum in: Oncologist 2000;5(5):following 438. Coney, K [corrected to Cooney, K]

2. Germ Cell Tumors of the Brain - Dana-Farber/Boston Children's Cancer and Blood Disorders Center [Internet] [cited 2019 Oct 16] Available from: http://www.danafarberbostonchildrens.org/conditions/brain-tumor/ germ-cell-tumors-of-the-brain.aspx

3. Wagner T. Ifosfamide clinical pharmacokinetics. Clin Pharmacokinet. 1994;26:439-56 [cited 2019 Oct 16] Available from: http://www.ncbi.nlm. nih.gov/pubmed/8070218.

4. Fleming RA. An overview of cyclophosphamide and ifosfamide pharmacology. Pharmacotherapy 17: 146S-154S. [cited 2019 Oct 16] Available from: http://www.ncbi.nlm.nih.gov/pubmed/9322882.

5. Ifosfamide neurotoxicity and potential treatment with methylene blue [Internet] [cited 2019 Oct 16] Available from: https://www.healio.com/ hematology-oncology/gynecologic-cancer/news/print/hemonctoday/\%7B9619000a-c6b7-4108-b02b-520e3233511c\%7D/ifosfamideneurotoxicity-and-potential-treatment-with-methylene-blue

6. Filhon B, Lacarra B, Hervouet C, Jaffray M, Schneider P, Vannier JP. Ifosfamide-induced encephalopathy due to a novel formulation of ifosfamide. Pediatr Blood Cancer. 2016;63(2):372-3.https://doi.org/10.1002/pbc 25724.

7. Szabatura AH, Cirrone F, Harris C, McDonnell AM, Feng Y, Voit D, Neuberg D, Butrynski J, Fisher DC. An assessment of risk factors associated with ifosfamide-induced encephalopathy in a large academic cancer center. J Oncol Pharm Pract. 2015;21(3):188-93. https://doi.org/10.1177/10781 55214527143.

8. Oz M, Lorke DE, Hasan M, Petroianu GA. Cellular and molecular actions of Methylene Blue in the nervous system. Med Res Rev. 2011;31(1):93117. https://doi.org/10.1002/med.20177.

9. Küpfer A, Aeschlimann C, Cerny T. Methylene blue and the neurotoxic mechanisms of ifosfamide encephalopathy. Eur J Clin Pharmacol. 1996;50(4):249-52. https://doi.org/10.1007/s002280050102.

10. Pelgrims J, De Vos F, Van den Brande J, Schrijvers D, Prové A, Vermorken JB. Methylene blue in the treatment and prevention of ifosfamideinduced encephalopathy: report of 12 cases and a review of the literature. Br J Cancer. 2000;82(2):291-4. https://doi.org/10.1054/bjoc.1999. 0917.

11. Park IS, Lee HJ, Lee YS, Hwang JS, Lee MS. Ifosfamide-induced encephalopathy with or without using methylene blue. Int J Gynecol Cancer. 2005;15(5):807-10. https://doi.org/10.1111/j.1525-1438.2005.00140.x.

12. MacDonald S, Liu K, et al. Neuro-Oncology. 2020;22(Suppl 3):iii335.

13. Skalny A, GeirBjørklund, et al. The role of glutathione redox imbalance in autism spectrum disorder: a review. Free Radic Biol Med. 2020;160:149-62.

14. Knouzy B, Dubourg L, Baverel G, Michoudet C. Targets of chloroacetaldehyde-induced nephrotoxicity. Toxicol In Vitro. 2010;24(1):99-107. https:// doi.org/10.1016/j.tiv.2009.08.026.

15. Wan Seok Seo. An update on the cause and treatment of sleep distrubance in childrena dna dolescents with autism spectrum disorder. Yeungnam Univ J Medicine. 2021;38(4):275-81.

16. Patel PN. Methylene blue for management of Ifosfamide-induced encephalopathy. Ann Pharmacother. 2006;40(2):299-303. https://doi.org/ 10.1345/aph.1G114.

17. Turner AR, Duong CD, Good DJ. Methylene blue for the treatment and prophylaxis of ifosfamide-induced encephalopathy. Clin Oncol. 2003.
18. Chloroacetaldehyde in The MAK-Collection for Occupational Health and Safety. Weinheim: Wiley-VCH Verlag GmbH \& Co. KGaA; 2012. pp 82-102. cited 2020 Nov 11. https://doi.org/10.1002/3527600418.mb10720e0012

\section{Publisher's Note}

Springer Nature remains neutral with regard to jurisdictional claims in published maps and institutional affiliations.
Ready to submit your research? Choose BMC and benefit from:

- fast, convenient online submission

- thorough peer review by experienced researchers in your field

- rapid publication on acceptance

- support for research data, including large and complex data types

- gold Open Access which fosters wider collaboration and increased citations

- maximum visibility for your research: over 100M website views per year

At BMC, research is always in progress.

Learn more biomedcentral.com/submissions 